\title{
\%, A CYTOPLASMIC SUPPRESSOR OF SUPER-SUPPRESSOR IN YEAST
}

\author{
B. S. $\operatorname{cox}$ \\ Deportment of Genetics, University of Liverpool
}

Received 24.ii.65

\section{INTRODUCTION}

IN 1963, Hawthorne and Mortimer reported an analysis of some suppressors of auxotrophic requirements in yeast. They found that such suppressors were unspecific in the sense that the suppression effect was not dependent on the function affected by a mutation, but were specific in that not all, only a proportion of the alleles leading to any particular loss of function were suppressible. Manney (1964) has confirmed that about a third of all ultra-violet induced mutants of yeast are suppressible by such suppressors. It appears from the data that separately isolated suppressors are often inherited independently of one another, and that, whenever tested, they have the same specificity.

During the course of studies on spontaneous inter-allelic reversions during vegetative growth in yeast, a number of revertants were recovered whose behaviour, on analysis, showed that they were due to genetically controlled suppression of one of the alleles in the diploid. A typical segregation, that of the revertant $Q_{5}$, is shown in table $I$. The original diploid used in the cross was composed as follows:

$$
2 n=\frac{a,}{\alpha} \frac{a d_{2,1}}{a d_{2, c}} \frac{h i_{8}}{+} \frac{s e_{1},}{+} \frac{m e_{2}}{+} \frac{t r_{1}}{+}=\text { red, adenine-requiring. }
$$

All markers except adenine requirement and colour segregate $2+: 2-$ in every tetrad. The adenine requirement, however, segregates $1+: 3$ - in four tetrads and o+: $4-$ in two tetrads. It is possible to distinguish $a d_{2,1}$ from $a d_{2}$, c by a complementation test (Woods, 1963). When this is done it is seen that $a d_{2,0}$ and $a d_{2,1}$ segregate regularly each to two spores in every tetrad. It is clear that the suppressor is specific for the $a d_{2,1}$ allele and does not affect $a d_{2, \text { o }}$ or any other marker. However it is dominant, for the diploid was white, and this affords a simple means of detecting its presence when it is coupled with $a d_{2, c}$ : the red strain " $a d_{2, c}, S U-a d_{2,1}$ " when mated with a red " $a d_{2,1}$ " will form white diploid cells, whereas " $a d_{2, ~} \times a d_{2,1}$ " is red. This test can also be used, of course, to identify the $a d_{2,1}$ allele, which is not a complementing mutant. Applying the test shows that the suppressor segregates regularly to two spores of every tetrad. This suppressor, now called SUQ 5, is the one appearing in all the experiments to be described here. 
In the course of the tetrad analysis of various crosses involving $S U Q_{5}$, a few cultures grown from ascospores were obtained which were impure. These were white cultures with red sectors or red cultures with prominent white sectors. The crosses concerned are set out in table 2. The experiments to be described involve cells isolated from

TABLE I

The segregation of markers in the white revertant $Q_{5}$

\begin{tabular}{|c|c|c|c|c|c|c|c|c|c|}
\hline $\begin{array}{c}\text { Tetrad } \\
\text { no. }\end{array}$ & Spore & $\begin{array}{c}1 \\
\text { Mating } \\
\text { type }\end{array}$ & 2 & 3 & 4 & 5 & $\mathrm{ad}_{2}$ allele & 7 & $\begin{array}{c}8 \\
\text { Colour }\end{array}$ \\
\hline 1 & $\begin{array}{l}a \\
b \\
c \\
d\end{array}$ & $\begin{array}{l}a \\
\alpha \\
\alpha \\
a\end{array}$ & $\begin{array}{c}+ \\
h i \\
h i \\
+\end{array}$ & $\begin{array}{l}+ \\
\text { se } \\
+ \\
\text { se }\end{array}$ & $\begin{array}{l}m e \\
+ \\
+ \\
m e\end{array}$ & $\begin{array}{l}t r \\
+ \\
+ \\
t r\end{array}$ & $\begin{array}{l}2 c \\
2 x \\
2 c \\
2 x\end{array}$ & $\begin{array}{l}+ \\
S U \\
S U \\
+\end{array}$ & $\begin{array}{l}\text { red } \\
\text { white } \\
\text { red } \\
\text { red }\end{array}$ \\
\hline 2 & $\begin{array}{l}a \\
b \\
c \\
d\end{array}$ & $\begin{array}{l}\alpha \\
a \\
\alpha \\
a\end{array}$ & $\begin{array}{c}+ \\
h i \\
h i \\
+\end{array}$ & $\begin{array}{l}+ \\
\text { se } \\
\text { se } \\
+\end{array}$ & $\begin{array}{c}m e \\
+ \\
m e \\
+\end{array}$ & $\begin{array}{l}t r \\
+ \\
+ \\
t r\end{array}$ & $\begin{array}{l}2 c \\
2 I \\
2 I \\
2 c\end{array}$ & $\begin{array}{l}+ \\
S U \\
+ \\
S U\end{array}$ & $\begin{array}{l}\text { red } \\
\text { white } \\
\text { red } \\
\text { red }\end{array}$ \\
\hline 3 & $\begin{array}{l}a \\
b \\
c \\
d\end{array}$ & $\begin{array}{l}\alpha \\
\alpha \\
a \\
a\end{array}$ & $\begin{array}{c}+ \\
h i \\
h i \\
+\end{array}$ & $\begin{array}{l}+ \\
\text { se } \\
\text { se } \\
+\end{array}$ & $\begin{array}{c}m e \\
+ \\
+ \\
m e\end{array}$ & $\begin{array}{l}\text { tr } \\
t r \\
+ \\
+\end{array}$ & $\begin{array}{l}2 c \\
2 I \\
2 C \\
2 I\end{array}$ & $\begin{array}{l}S U \\
+ \\
S U \\
+\end{array}$ & $\begin{array}{l}\text { red } \\
\text { red } \\
\text { red } \\
\text { red }\end{array}$ \\
\hline 4 & $\begin{array}{l}a \\
b \\
c \\
d\end{array}$ & $\begin{array}{l}a \\
a \\
\alpha \\
\alpha\end{array}$ & $\begin{array}{l}h i \\
+ \\
+ \\
h i\end{array}$ & $\begin{array}{l}+ \\
\text { se } \\
+ \\
\text { se }\end{array}$ & $\begin{array}{c}m e \\
+ \\
+ \\
m e\end{array}$ & $\begin{array}{l}\text { tr } \\
+ \\
t r \\
+\end{array}$ & $\begin{array}{l}2 I \\
2 I \\
2 c \\
2 c\end{array}$ & $\begin{array}{l}S U \\
+ \\
+ \\
S U\end{array}$ & $\begin{array}{l}\text { white } \\
\text { red } \\
\text { red } \\
\text { red }\end{array}$ \\
\hline 5 & $\begin{array}{l}a \\
b \\
c \\
d\end{array}$ & $\begin{array}{l}\alpha \\
a \\
\alpha \\
a\end{array}$ & $\begin{array}{l}h i \\
+ \\
+ \\
h i\end{array}$ & $\begin{array}{l}\text { se } \\
+ \\
+ \\
\text { se }\end{array}$ & $\begin{array}{c}+ \\
+ \\
m e \\
m e\end{array}$ & $\begin{array}{l}\text { tr } \\
+ \\
+ \\
t r\end{array}$ & $\begin{array}{l}2 I \\
2 I \\
2 c \\
2 c\end{array}$ & $\begin{array}{l}+ \\
+ \\
S U \\
S U\end{array}$ & $\begin{array}{l}\text { red } \\
\text { red } \\
\text { red } \\
\text { red }\end{array}$ \\
\hline 6 & $\begin{array}{l}a \\
b \\
c \\
d\end{array}$ & $\begin{array}{l}a \\
\alpha \\
a \\
\alpha\end{array}$ & $\begin{array}{l}h i \\
+ \\
+ \\
h i\end{array}$ & $\begin{array}{l}\text { se } \\
+ \\
+ \\
\text { se }\end{array}$ & $\begin{array}{l}+ \\
+ \\
m e \\
m e\end{array}$ & $\begin{array}{l}t r \\
+ \\
t r \\
+\end{array}$ & $\begin{array}{l}2 \mathrm{I} \\
2 c \\
2 c \\
2 \mathrm{I}\end{array}$ & $\begin{array}{l}+ \\
S U \\
+ \\
S U\end{array}$ & $\begin{array}{l}\text { red } \\
\text { red } \\
\text { red } \\
\text { white }\end{array}$ \\
\hline
\end{tabular}

the red and white portions of the three "impure cultures" in which the white portions were adenine independent.

\section{MATERIALS AND METHODS \\ (i) Strains}

All yeast strains used were derived from cultures provided at one time or another by $\mathrm{Dr} \mathrm{H}$. Roman or by $\mathrm{Dr} \mathrm{R}$. K. Mortimer. The $s e_{1}$ and all the adenine mutants were isolated by the author or by Woods, except $a d_{2}$, 1 , which is the original $a d_{2}$ allele described by Roman (Roman, 1956). This and all other mutants originate in Roman's or Mortimer's stocks. The abbreviations and symbols used throughout the text are those agreed on at the Carbondale Yeast Genetics conference in 1958. 


\section{(ii) Media}

The media used have been described previously (Cox and Bevan, 1962). The complete medium used is a yeast extract/peptone medium containing 4 per cent. of glucose. All $a d_{2}$ mutants are red or pink when grown on it. The synthetic minimal

TABLE 2

Diploids giving rise to impure cultures from ascospores

\begin{tabular}{|c|c|c|c|c|c|c|}
\hline \multirow{2}{*}{$\begin{array}{l}\text { Diploid } \\
\text { no. }\end{array}$} & \multirow{2}{*}{ Genotype } & \multirow{2}{*}{\multicolumn{2}{|c|}{$\begin{array}{l}\text { Segregation } \\
\text { of markers }\end{array}$}} & \multirow{2}{*}{$\begin{array}{l}\text { Tetrad containing } \\
\text { an "impure" } \\
\text { culture }\end{array}$} & \multicolumn{2}{|c|}{$\begin{array}{l}\text { Adenine } \\
\text { requirement of }\end{array}$} \\
\hline & & & & & $\underset{\text { sector }}{\text { Red }}$ & $\begin{array}{l}\text { White } \\
\text { sector }\end{array}$ \\
\hline 159 & $\frac{\alpha}{a} \frac{a d_{21}}{a d_{2-r d}} \frac{S U Q_{5}}{+} \frac{t r}{+}$ & $\begin{array}{l}\text { adenine } \\
\text { tryptophan }\end{array}$ & $\begin{array}{l}2: 2 \\
2: 2\end{array}$ & $\begin{array}{l}\text { 7a red } \\
7 \mathrm{~b} \text { red } \\
7 \mathrm{c} \text { red with white } \\
\text { sector } \\
7 \mathrm{~d} \text { white }\end{array}$ & $a d^{-}$ & $a d^{-}$ \\
\hline 162 & $\frac{\alpha}{a} \frac{a d_{21}}{a d_{2-r 00}} \frac{S U Q_{5}}{+} \frac{t r}{+}$ & $\begin{array}{l}\text { adenine } \\
\text { tryptophan }\end{array}$ & $\begin{array}{l}2: 2 \\
2: 2\end{array}$ & $\begin{array}{l}8 \mathrm{a} \text { red } \\
8 \mathrm{~b} \text { red } \\
8 \mathrm{c} \text { red with white } \\
\text { sector } \\
8 \mathrm{~d} \text { white }\end{array}$ & $a d^{-}$ & $a d^{-}$ \\
\hline 163 & $\frac{\alpha}{a} \frac{a d_{21}}{a d_{2-r 158}} \frac{S U Q_{5}}{+} \frac{t r}{t}$ & $\begin{array}{l}\text { adenine } \\
\text { tryptophan }\end{array}$ & $\begin{array}{l}2: 2 \\
2: 2\end{array}$ & $\begin{array}{l}\text { 9a red } \\
\text { gb white } \\
\text { gc white with red } \\
\text { sector } \\
\text { 9d red }\end{array}$ & $a d^{-}$ & $a d^{+}$ \\
\hline 170 & $\frac{\alpha}{a} \frac{a d_{21}}{a d_{21}} \frac{S U Q_{5}}{S U Q_{5}} \frac{t r}{+}$ & $\begin{array}{l}\text { adenine } \\
\text { tryptophan }\end{array}$ & $\begin{array}{l}4: 0 \\
2: 2\end{array}$ & $\begin{array}{l}2 \mathrm{a} \text { white } \\
2 \mathrm{~b} \text { white } \\
2 \mathrm{c} \text { red with white } \\
\text { sector } \\
2 \mathrm{~d} \text { white }\end{array}$ & $a d^{-}$ & $a d^{+}$ \\
\hline 172 & $\frac{\alpha}{a} \frac{a d_{21}}{a d_{21}} \frac{S U Q_{5}}{S U Q_{5}} \frac{t r}{t}$ & $\begin{array}{l}\text { adenine } \\
\text { tryptophan }\end{array}$ & $\begin{array}{l}4: 0 \\
2: 2\end{array}$ & $\begin{array}{l}9 \text { a white } \\
9 \text { b white with red } \\
\text { sector } \\
9 \mathrm{c} \text { white } \\
9 \mathrm{~d} \text { white }\end{array}$ & $a d^{-}$ & $a d^{+}$ \\
\hline
\end{tabular}

medium used was either that previously described, or Difco Yeast Nitrogen Base without amino-acids, solidified with Oxoid Ionagar No. 2. Fowell's sporulation medium (Fowell, 195I) was used to obtain asci in diploid cultures.

\section{(iii) Methods}

Standard bacteriological techniques were used in plating and subculturing. Unless otherwise stated, all diploids were formed by isolating single zygotes from a mating mixture using a micromanipulator. Asci were dissected for tetrad analysis by Johnston and Mortimer's method (Johnston and Mortimer, 1959). Auxotrophic requirements were scored by replica-plating onto suitably supplemented minimal 
media. Adenine requirement was routinely tested, regardless of the colour of the culture. There were few exceptions to the rule that in these strains, adenine requirers are red and non-requirers white. The exceptions are made clear in the text. $a d_{2}$ allelles were distinguished from each other by complementation tests (Woods, 1963).

\section{EXPERIMENTS AND RESULTS}

(I) Stability of red and white portions of impure cultures

Purified sub-cultures of the red and white portions of the three cultures were plated and streaked on complete medium, and the cultures examined with a low-power microscope for sectors of opposite colour. The results are set out in table 3. A number of the white sectors arising on red cultures spontaneously, were tested for auxotrophic requirements and proved to be adenine independent. The

TABLE 3

Stability of red and white cultures

\begin{tabular}{|c|c|c|}
\hline Strain & Red culture & White culture \\
\hline $163 / 9 c$ & $\begin{array}{l}\text { Several pink sectors, a very } \\
\text { few white papillz }\end{array}$ & Stable \\
\hline $170 / 2 \mathrm{c}$ & A few white papille & Stable \\
\hline $172 / 9 b$ & A very few white papillx & $\begin{array}{l}\text { One red sector * in } \\
629 \text { colonies }\end{array}$ \\
\hline${ }^{*} 172 / 9 b$ red sector & A few white papilla & $\ldots$ \\
\hline
\end{tabular}

nature of the reversions is being investigated. In general, the rate of reversion is extremely low and is comparable to normal mutation rates.

\section{(ii) Intercrosses of red cultures}

The following diploids were synthesised by mating single cells and isolating the zygotes:

$$
\begin{aligned}
& 163 / 9 c \mathrm{red} \times 170 / 2 c \mathrm{red} \\
& 172 / 9 b \mathrm{red} \times 170 / 2 c \mathrm{red} \\
& 172 / 9 b \mathrm{red} \text { sector } \times 170 / 2 c \mathrm{red} .
\end{aligned}
$$

All three diploids were red adenine requirers. This is taken to indicate that the functional losses are identical in the three strains.

Ascospores were dissected for a tetrad analysis. In the first cross, $2 n=163 / 9 c \mathrm{red} \times 170 / 2 c \mathrm{red}$, germination on complete medium was very poor ( 5 per cent.) but on minimal supplemented with adenine, improved to 40 per cent. All the cultures which grew from ascospores were red, and all were most unstable. The instability typically takes the form of the dark red cultures throwing frequent pink and white sectors. The purified pink sectors throw dark red and white sectors, and purified white sectors throw a very few pink sectors which are 
then able to throw dark red and white sectors again. Dark red and pink cultures require adenine, white cultures do not. The nature of this instability, which is a common characteristic of cytoplasmic change (see Jinks, 1964), is under investigation.

In the second cross, 77.5 per cent. of ascospores germinated. All were red adenine requirers, and were stable. Two of the spore cultures were backcrossed to the parent cultures. The diploids were red adenine requirers and one, after ascospore analysis, produced entirely red spore cultures. The back-crosses of two of the segregants to the parent cultures indicate that the mutation is inherited unchanged after sexual reproduction. The crosses and the results are summarised

TABLE 4

Results of intercrossing red cultures from "impure" strains

\begin{tabular}{|c|c|c|c|c|c|c|}
\hline \multirow{2}{*}{ Cross } & \multirow{2}{*}{ No. } & \multirow{2}{*}{$\begin{array}{l}\text { Diploid } \\
\text { colour }\end{array}$} & \multirow{2}{*}{ Requirement } & \multirow{2}{*}{ Germination } & \multicolumn{2}{|c|}{ Ascospores } \\
\hline & & & & & Colour & Requirement \\
\hline $\begin{array}{l}\text { (a) Intercrosses: } \\
170 / 2 \mathrm{c}, \mathrm{red} \times 163 / 9 \mathrm{cred} \\
170 / 2 \mathrm{cred} \times 172 / 9 \mathrm{~b} \text { red } \\
170 / 2 \mathrm{cred} \times 172 / 9 \mathrm{~b} \text { red } \\
\text { sector }\end{array}$ & $\begin{array}{l}194 \\
193 \\
\cdots\end{array}$ & $\begin{array}{l}\text { red } \\
\text { red } \\
\text { red }\end{array}$ & $\begin{array}{l}\mathrm{ad}^{-} \\
\mathrm{ad}^{-} \\
\mathrm{ad}^{-}\end{array}$ & $\begin{array}{c}40 \text { per cent. } \\
77 \cdot 5 \text { per cent. } \\
\cdots\end{array}$ & $\begin{array}{l}\text { red } \\
\text { all red } \\
\text { very po }\end{array}$ & $\begin{array}{c}a^{-} \text {(unstable) } \\
\text { ad- } \\
\text { or sporulation }\end{array}$ \\
\hline $\begin{array}{l}\text { (b) Back-crosses: } \\
170 / 2 \mathrm{red} \times 193 / 1 \mathrm{a} * \\
172 / 9 \mathrm{~b} \text { red } \times 193 / 1 \mathrm{~b}\end{array}$ & $\begin{array}{l}199 \\
\ldots\end{array}$ & $\begin{array}{l}\text { red } \\
\text { red }\end{array}$ & $\begin{array}{l}\mathrm{ad}^{-} \\
\mathrm{ad}^{-}\end{array}$ & $\begin{array}{c}70 \cdot 0 \text { per cent. } \\
\ldots\end{array}$ & $\begin{array}{c}\text { all red } \\
\ldots\end{array}$ & $\begin{array}{l}a d^{-} \\
\cdots\end{array}$ \\
\hline
\end{tabular}

*These are red spore-cultures from the diploid $193=170 / 2 \mathrm{cred} \times 172 / 9 \mathrm{~b}$ red (this table, line 2 ).

in table 4. It is clear that the red colour and adenine requirement in these strains is stably inherited through both vegetative and sexual reproduction.

\section{(iii) Outcrosses of red and white cultures}

The white cultures of the "impure" strains were all crossed to haploid wild-type yeast. The diploids were white and adenine independent. Spore germination in two of the diploids was very poor. The results are shown in table 5 .

The segregation of tetrads of diploid I 75 (line 2) is that expected from a diploid heterozygous for both adenine requirement and a suppressor of adenine requirement. The simplest explanation of the phenotypes segregating from the other diploids is the same, so it is concluded that all three white cultures derived from impure strains have the genotype: $a$ or $\alpha, a d_{2,1}, S U Q_{5}$, that is, that they contain the suppressor present in the diploids from which they are derived.

The three red portions of the "impure" cultures and the four cultures from one tetrad of a diploid formed by mating two of them, 
were crossed with both suppressed and unsuppressed $a d_{2,1}$ strains. The results are set out in table 6 .

TABLE 5

Results of crossing white cultures with wild-type haploid yeast

\begin{tabular}{|c|c|c|c|c|}
\hline \multirow{2}{*}{ Cross } & \multirow{2}{*}{ No. } & \multicolumn{2}{|c|}{ Diploid } & \multirow{2}{*}{ Ascospores and tetrads } \\
\hline & & Colour & Requirement & \\
\hline $\mathrm{WT} \times 16_{3} / 9 c$ white & 187 & White & $a d^{+}$ & $\begin{array}{l}5 \text { white adenine independent spore- } \\
\text { cultures } \\
\text { I red adenine requiring spore- } \\
\text { culture }\end{array}$ \\
\hline $\mathrm{WT} \times 170 / 2 c$ white & I 75 & White & $a d+$ & $\begin{array}{l}\text { I tetrad with } 4 \text { ad }+ \text { : o ad- spore- } \\
\text { cultures } \\
6 \text { tetrads with } 3 \text { adt : I ad- spore- } \\
\text { cultures }\end{array}$ \\
\hline $\mathrm{WT} \times 172 / 9 b$ white & 185 & White & $a d+$ & $\begin{array}{l}5 \text { white adenine independent spore- } \\
\text { cultures } \\
4 \text { red adenine requiring spore- } \\
\text { cultures }\end{array}$ \\
\hline
\end{tabular}

The diploid phenotypes and segregations to be expected from these crosses depend upon the genotype of the red portions of the impure cultures. We may first consider two possibilities. One is

TABLE 6

Results of crossing red cultures with unsuppressed and suppressed $\mathrm{ad}_{2,1}$ mutants of yeast

\begin{tabular}{|c|c|c|c|c|}
\hline \multirow{2}{*}{ Strain } & \multicolumn{2}{|c|}{$\begin{array}{l}\text { Crossed with unsuppressed red } \\
\qquad a d_{2,1}\end{array}$} & \multicolumn{2}{|c|}{$\begin{array}{l}\text { Crossed with suppressed white } \\
\qquad a d_{2,1} S U Q_{5}\end{array}$} \\
\hline & Diploid & $\begin{array}{l}\text { No. of tetrads with } \\
2 A D+: 2 \mathrm{ad}^{-}\end{array}$ & Diploid & $\begin{array}{l}\text { No. of tetrads with } \\
4 A D+: 0 \mathrm{ad}^{-}\end{array}$ \\
\hline $\begin{array}{l}\text { 1. } 163 / 9 c \mathrm{red} \\
\text { 2. } 170 / 2 c \mathrm{red} \\
\text { 3. } 172 / 9 b \mathrm{red} \\
\text { 4. } 193 / 1 a^{*} \\
\text { 5. } 193 / 1 b^{*} \\
\text { 6. } 193 / 1 c^{*} \\
\text { 7. } 193 / 1 d^{*}\end{array}$ & $\begin{array}{l}A D+, \text { pink } \\
A D+, \text { white } \\
A D+, \text { white } \\
A D+, \text { pink } \\
A D+, \text { pink } \\
A D+, \text { pink } \\
A D+, \text { pink }\end{array}$ & $\begin{array}{r}8 \\
4 \\
5 \\
12 \\
5 \\
2 \\
7\end{array}$ & $\begin{array}{c}A D+, \text { white } \\
A D+, \text { white } \\
A D+, \text { white } \\
\ldots \\
\ldots \\
\ldots \\
\ldots\end{array}$ & $\begin{array}{c}\text { very poor sporulation } \\
9 \\
7 \\
\ldots \\
\cdots \\
\ldots \\
\ldots\end{array}$ \\
\hline
\end{tabular}

* These are the four cultures from one tetrad of the diploid $193=170 / 2 c$ red $\times$ ${ }^{7} 72 / 9^{b}$ red. All four were red, $a d^{-}$(table 4 , line 2 ).

that these strains are wild-type at the $S U Q_{5}$ locus, through backmutation of $S U Q_{5}$. In this case the genotype of the red strains would be " $a d_{2,1}$ ". The second is that a further mutation has occurred suppressing the suppressor, $S U Q_{5}$. The red genotype would then be 
" $a d_{2,1}, S U Q_{5}, S U-S U$ ". If strains of either of these genotypes were to be crossed with a white strain of genotype " $a d_{2,1}, S U Q_{5}$ ", the tetrads derived after meiosis would all give rise to two red and two white cultures, due to the segregation of the one gene for which each diploid is heterozygous. (SUQ 5 in the first case, $S U-S U$ in the second). In neither of the tetrad analyses carried out (table 9, lines 2 and 3 ) has this occurred. Instead, all sixteen tetrads gave four white adenine non-requiring cultures.

Similarly the matings with unsuppressed $a d_{2,1}$ strains do not give results expected from either of the possible genotypes mentioned. If the de-suppressed reds are of genotype " $a d_{2,1}$ ", then the diploid formed would of course be homozgyous at this locus, $2 n=a d_{2,1} / a d_{2,1}$, and the tetrads would all give four red cultures. If the second possible genotype is considered, crossing with an unsuppressed red would give a diploid heterozygous at two loci-SU 5 and $S U-S U\left(2 n=a d_{2,1} / a d_{2,1}\right.$, $S U Q 5 /+, S U-S U /+)$. The tetrads would be of three classes: parental ditypes with four red cultures $(0: 4)$; non-parental ditypes with two red and two white cultures $(2: 2)$ and tetratypes with three red and one white culture $(1: 3)$. Linkage of the second suppressor to the first would increase the frequency of $0: 4$ ratios and decrease the other two classes, especially the $2: 2$ ratios (Perkins, 1953). In fact, all the forty-three tetrads from seven diploids segregate 2 white $: 2$ red cultures.

All these segregations, and also the phenotypes of the diploids formed by the various matings, are exactly those expected if the desuppressed red strains, like the white portions of the colonies in which they arose, are of genotype $a d_{2,1}, S U Q_{5}$. It must be concluded that the suppressor is present in all these red strains but is inactive; that the inactivity is a permanent condition provided the strains are not mated, but once they are mated with normal strains, the suppressor regains its activity. The inactive state is never recovered by segregation, therefore it is not due to the mutation of a chromosomal gene, and must be due to a cytoplasmic mutation.

\section{(iv) The effect of the suppressor SUQ5 on genes suppressible by super-suppressor}

A haploid strain of yeast was provided by $\operatorname{Dr} \mathrm{R}$. K. Mortimer which contained the following markers:

$$
\mathcal{J} M_{32}=a, a d_{2,1}, h i_{5,2}, l y_{1,1}, a r_{4,23} .
$$

$a d_{2,1}, h i_{5,2}$ and $l y_{1,1}$ are all suppressible by super-suppressors, while $a r_{4,23}$ is not (Mortimer, personal communication). The results of crossing this strain with strains of various genotypes are set out in table 7. It is clear from the segregation of requirements in the first two crosses that the suppressor present in these crosses suppresses $h i_{5,2}$ and $l y_{1,1}$ as well as $a d_{2,1}$, and does not suppress $a r_{4,23}$. This is 
B. S. COX

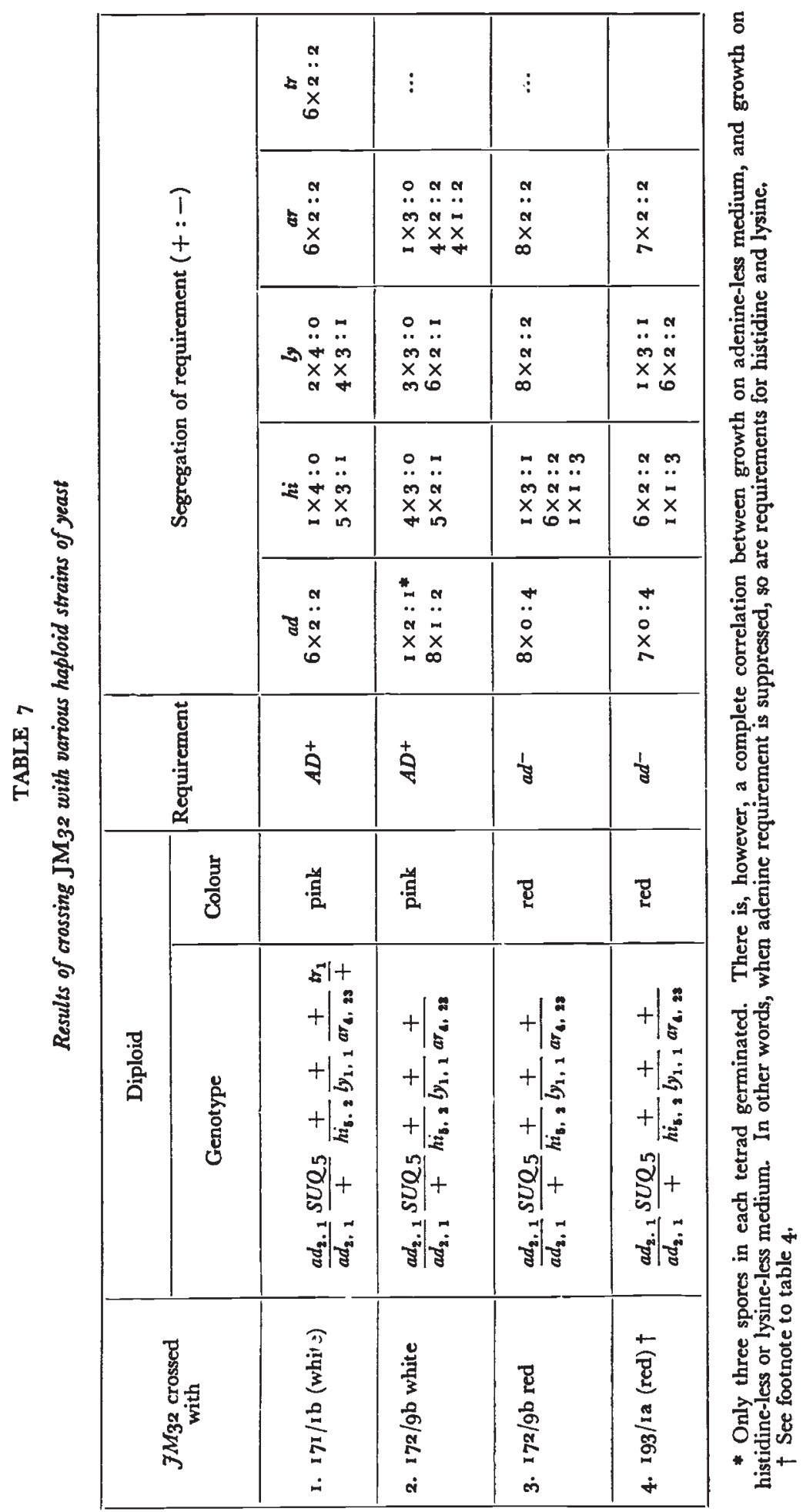


even more obvious when the detailed results are studied, for these show an exact correlation between the ability of segregants to grow without adenine and their ability to grow without histidine or lysine. It may therefore be concluded that the suppressor, $S U Q_{5}$ isolated and described here falls into the class of "super-suppressors " described by Hawthorne and Mortimer.

The results from the second two crosses are unexpected. It will be recalled that in the previous outcrosses of de-suppressed red strains in which the super-suppressor gene was present but inactive (table 6), the suppressor became active again, both in the diploids synthesised and their spore-progeny. In these later outcrosses, however, it is clear that the suppressor, if present, is still inactive, since the diploid cultures and all their spore progeny are red adenine requirers, and histidine and lysine requirement, with a few exceptions both segregate $2+: 2-$. In order to determine whether the suppressor is present and segregating in these diploids, a tetrad from one of them, diploid $197=2 n=193 / 1 a \operatorname{red} \times \mathcal{J} M_{32}$ (table 7 , line 4 ), was taken and back-crossed to suppressed and unsuppressed $a d_{2,1}$ strains. The results are set out in table 8 .

It is clear from the segregations and from the phenotypes of the synthesised diploids that two of the cultures in this tetrad, 197/ $a$ and $197 / 1 d$, contain the super-suppressor, while the other two do not. It is concluded that the super-suppressor is present, but inactive in the parental red strain, $193 / \mathrm{r} a$, in the diploid synthesised with $\mathcal{F M}_{32}$ and among the spore-progeny of the diploid. Evidence that the suppressor segregates in a normal Mendelian fashion comes from cross-streaking the spore-cultures from tetrads of diploid 197 with $a d_{2,1}$ mutant strains of opposite mating-type. Two cultures from each tetrad invariably gave white or pink growth where the dark red streaks crossed each other, while the other two showed no different colouration at all.

The possibility remains that the inactivity of the suppressor in these outcrosses is a chance property of one of the cells which contribute to the single zygote from which the diploid culture is grown. To see whether there is any variation among the cells of either parental culture in this respect, mass-matings were carried out by Lindegren's technique (Lindegren, 1949), that is without selection of any zygotes. The two cultures are simply mixed on complete agar medium, allowed to grow together for two days and then transferred to sporulation medium. When asci are taken for dissection from such mixtures, it may fairly be presumed that they all originate from different zygotes. The crosses repeated were $193 / \mathrm{r} a \times a d_{2,1}$ and $193 / \mathrm{r} a \times 7 M_{32}$ (table 6 , line 4 , and table 7 , line 4). As far as the segregation of adenine requirement is concerned, the results were identical with those from the single-zygote diploid cultures, and unambiguous. In the first cross, both colour and requirement segregated $2: 2$ (five tetrads). In the second cross, all the segregants (6 tetrads) were adenine 


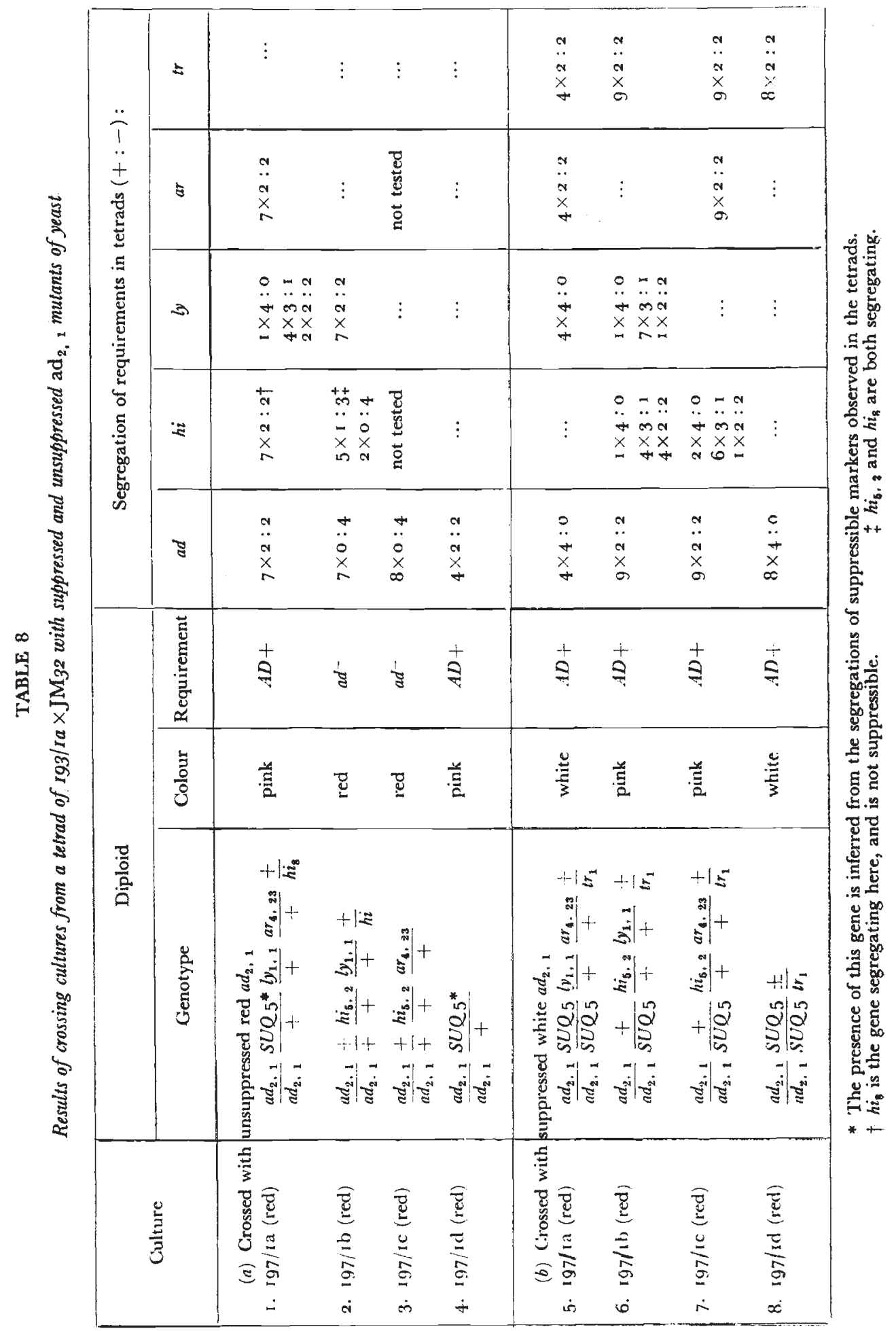


requirers. There was, however, some ambiguity over colour, since many of the cultures were a paler pink and one was white. It also appeared in the second mating that $h i_{5,2}$ was now being suppressed, though not always fully suppressed, and also, although a $2: 2$ segregation of lysine requirement could easily be scored, many of the "negative" cultures did, in fact, grow slightly: they showed "leaky" growth. In general, it may be concluded that, although there is evidence of variation among the cells of one or other parent in the degree to which super-suppressor remains inactive in the mass-matings, the overall effect is the same as in the original single-zygote crosses.

In order to determine whether the inactivity of the super-suppressor, which in the cross of $193 / 1 a \times \mathcal{F M}_{32}$ (diploid 197) survived one complete sexual cycle, would persist for another generation, one of the red segregants containing the super-suppressor was back-crossed to the parental strains. The results are set out in the first two lines of table 9 . In both cases, it is clear that the suppressor is still inactive in the diploid and its spore progeny as far as adenine requirement is concerned, but there are signs of suppression of histidine and lysine requirement in the form of leaky growth.

The last four lines of table 9 show the results of crossing all the four cultures from this tetrad of diploid 197 with the parental or parental-type strains containing an inactive super-suppressor. Of these four cultures, of course, two contain $S U Q_{5}$, while the other two do not (table 8). It is clear that all four segregants have the characteristic of the parent $\mathcal{J} M_{32}$ that they do not restore the supersuppressor to activity when crossed with " inactive super-suppressor" strains. Thus it appears that the inactivity of the super-suppressor can be maintained, in suitable crosses, through three sexual cycles. It may therefore be concluded that this inactive condition does not suffer dilution either through succeeding vegetative or sexual generations. A diagrammatic representation of the principal crosses and their results is given in fig. I. Before discussing them, the results may be summarised as follows.

(I) Some $a d_{2,1}$ mutant cultures of yeast which are expected, from their parentage, to be white by virtue of the presence of a supersuppressor suppressing the $a d_{2,1}$ allele, have a red, adenine-requiring phenotype which is stable.

(2) The red, adenine-requiring phenotype is retained when cultures of this kind, of independent origin are mated with each other, both in the diploids formed, their progeny and in back-crosses of the progeny (table 4 ).

(3) When outcrossed to suppressed $a d_{2,1}$ mutants of yeast, the red adenine-requiring phenotype fails to segregate in the progeny (table 6).

(4) When outcrossed to certain unsuppressed $a d_{2,1}$ mutants, the super-suppressor segregates in the progeny in a normal Mendelian fashion and produces a white non-requiring phenotype. There is no segregation of a de-suppressed red phenotype (table 6). 
B. S. COX

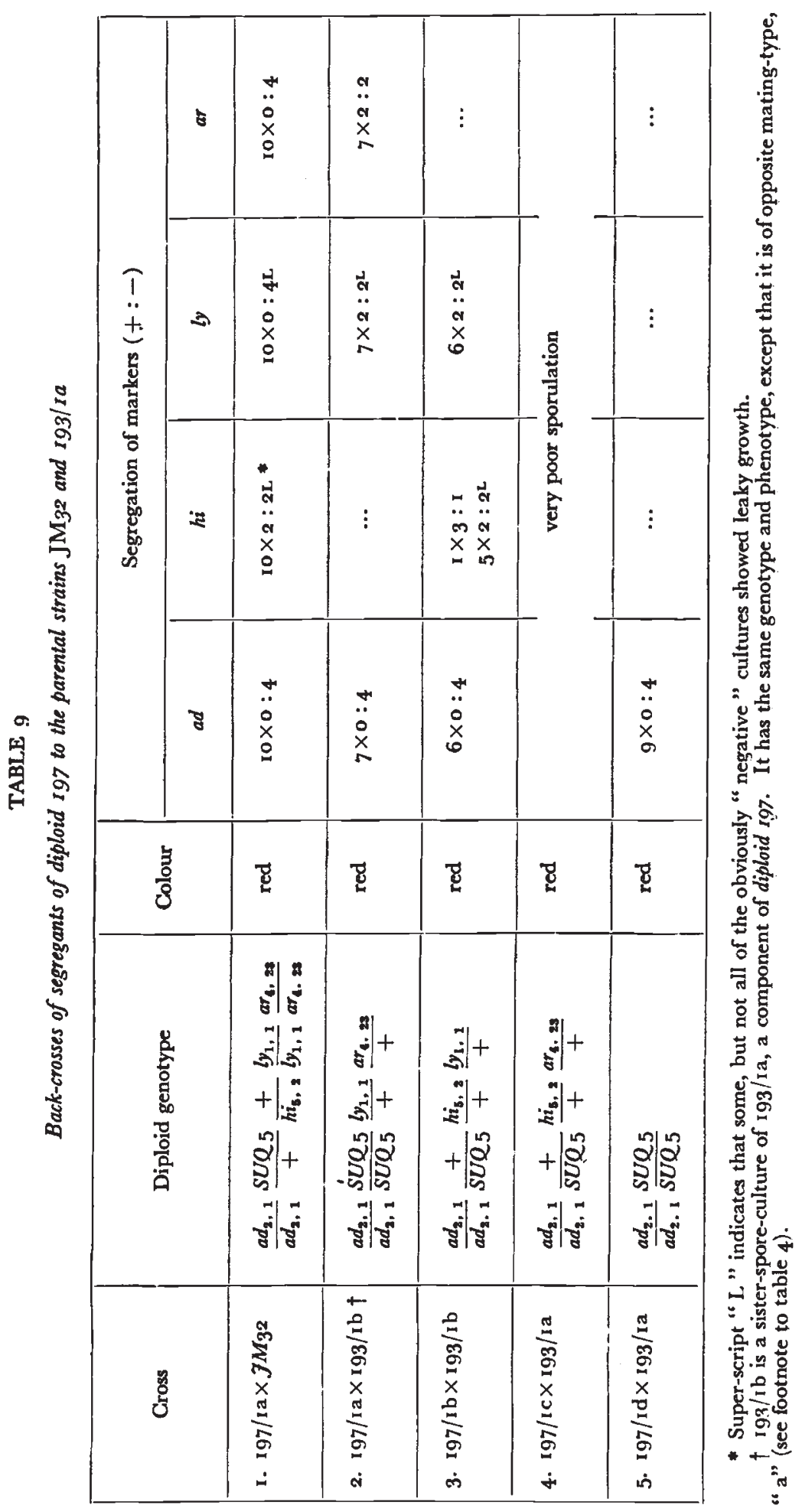


(5) When outcrossed to another unsuppressed $a d_{2,1}$ mutant $\left(\mathcal{J} M_{32}\right)$, no white non-requiring progeny segregate, and two other

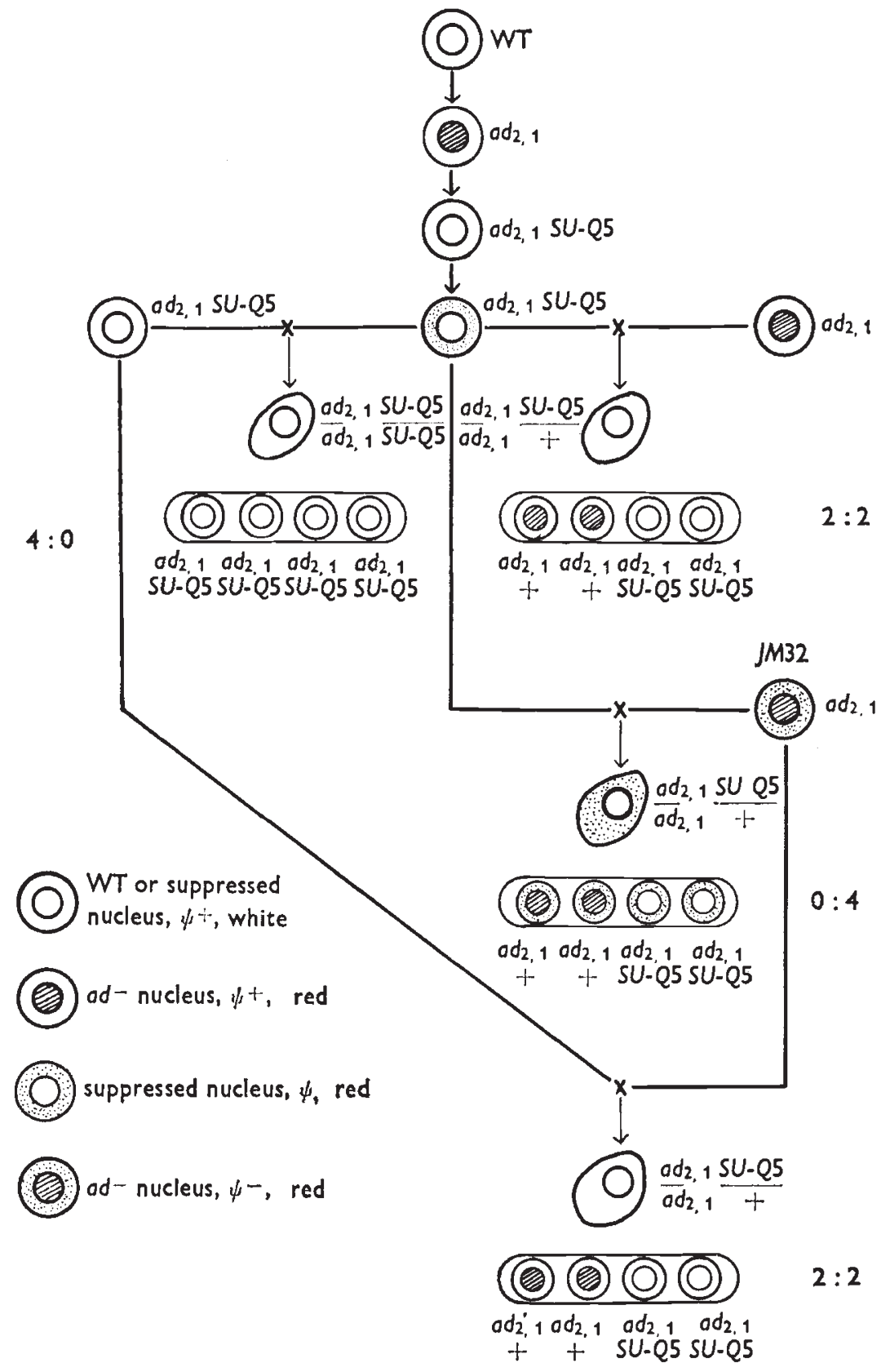

Fig. 1.-Summary of the principal crosses and their results.

alleles, $h i_{5,2}$ and $l y_{1,1}$, which are also suppressible by the supersuppressor, segregate normally (table 7). 
(6) Nevertheless, the super-suppressor can be shown to be present and segregating in a Mendelian fashion in these progeny by suitable outcrosses (table 8).

(7) These retain the property of their parental cultures in that in back-crosses the super-suppressor remains inactive.

\section{DISCUSSION}

The experiments show that a stable change of phenotype, or mutation, has taken place in a few strains of suppressed $a d_{2}, 1$ mutants of yeast, which renders the super-suppressor inactive. Four such mutations independently isolated are identical as judged by the fact that they fail to complement in diploids. The mutations are stable not only through vegetative, but through sexual reproduction. In certain outcrosses, however, activity is restored both in the diploid stage and in all the spore-progeny; in other words the mutation, or its effect disappears, and does not segregate in a Mendelian fashion. In other outcrosses, the inactivity persists, but again it persists in all the spore-progeny, and does not segregate in a Mendelian fashion, unless it segregates closely linked to the super-suppressor. This possibility is discounted by back-crosses of segregants.

The simplest explanation of these events is in terms of a stable cytoplasmic mutation which interferes with the action of the supersuppressor. We may suppose that the suppressed $a d_{2}, \mathbf{1}$ strains, which because of the super-suppressor have a white, adenine nonrequirer phenotype, contain a self-replicating particle, or a system of reactions sustaining itself by positive feed-back (Novick and Weiner, 1957), whose presence and activity are necessary for the supersuppressor activity to be expressed. In the red portions of the "impure" spore cultures, this particle is either absent, present in very low numbers, or has mutated so that the super-suppressor activity is no longer expressed. On crossing these red mutant strains with any " normal" strain, in this case with either red unsuppressed or white suppressed strains, a normal cytoplasm is restored and the supersuppressor phenotype is again expressed in the diploid, and segregates in the progeny. By analogy with those systems of cytoplasmic inheritance already well known in fungi, it might be supposed that in these outcrosses in which the mutation "disappears" never to be recovered, the cytoplasm of the mutant strains is " neutral" and the activity of the super-suppressor is restored by the "suppressive"* action of the normal cytoplasm. This is identical to the fate of the neutral petite phenotype $\left(\rho^{-}\right)$of yeast when it is crossed to wild-type

* The terminology at this point is liable to become confusing. A suppressor mutation is a mutation other than a back-mutation at the same site, which restores the wild-type phenotype to a mutant strain. The term suppressive on the other hand was coined (Darlington, 1944) to dcscribe the situation, common in examples of cytoplasmic inheritance in fungi (Jinks, 1063 ) in which the mixing of two different cytoplasms leads to the total disappearance of one of the phenotypes. It is, perhaps, analogous to dominance in Mendelian inheritance, although the " recessive" cytoplasmic character may never segregate. 
strains (Ephrussi, I 953). There are then two possible interpretations of the results of the outcrosses to $7 M_{32}$ in which the super-suppressor remains inactive. The simplest is that the strain used, $7 M_{32}$, is also mutant in the same way. This apparently has no effect on its phenotype, but as it contains no super-suppressor, such an effect might not be detectable. If it has a similar mutation, it could not be "suppressive" of the mutant cytoplasm. The second possibility is that it is not mutant in the same way, but has nevertheless a different cytoplasm from the " normal" strain used in the other outcrosses, a cytoplasm which is not suppressive of the mutant. The analogy with the chromosomal gene systems would be for the first alternative: two non-complementing allelic mutations; for the second: either three alleles with different levels of dominance interaction, one in "normal" strains, one in de-suppressed red strains, and the third allele in $7 M_{32}$; or two alleles, a mutant and a normal allele, whose interactions are affected by a modifier in $7 M_{32}$. This raises the possibility of a chromosomal rather than a cytoplasmic " modifier" to account for the reaction with $\mathcal{7} M_{32}$. The fact, however, that all four products of a single tetrad of the outcross to $\mathcal{J M}_{32}$ (197/1 a , $\mathrm{I} b$, Ic and $\mathrm{I} d$ ) behave similarly in back-crosses shows that no such modifier is segregating.

It is proposed to call the particle or system necessary for the expression of super-suppressor activity $\psi$. Normal strains in which this super-suppressor is active are $\psi^{+}$. The de-suppressed red strains are $\psi^{-}$.

It is interesting to consider the significance of the cytoplasmic suppressor of this super-suppressor in the light of what is known about the mode of action of super-suppressors. In doing so, though, it must be remembered that the identification of the suppressor mutation SUQ 5 with other super-suppressors is based on its effect on three mutant alleles only, and that the cytoplasmic suppressor of supersuppressor has not yet been tested with other super-suppressors. Hawthorne and Mortimer ( 1963 ) and Manney ( 1964 ) have considered several models for the action of super-suppressor, and these divide into three categories. One may be called the "enzyme level modifier" theory in which it is supposed that suppressible mutants are a class that produces a very small number of molecules of normal protein and that the suppressor acts to raise the general level of protein synthesis to a state where sufficient is produced even by suppressible mutant genes. The second model may be called the "sensitive protein" theory. Here it is supposed that the suppressible mutations govern the production of mutant proteins which are sensitive to a specific (e.g. ionic) or less specific (e.g. pH, temperature) inhibitor in the cell or its environment and that the suppressor either removes the inhibitor or stabilises the mutant proteins (cf. Campbell, I 96I; Suskind and Kurek, I959). The third model is the " nonsense code" theory. This supposes that the suppressible mutations are responsible 
for converting a meaningful sequence of the DNA code to a " nonsense" sequence. Benzer and Champe (1962) and Sarabhai et al. (r964) have shown in bacteriophage, that polypeptide synthesis ceases at the point at which a nonsense sequence appears in the genetic material, and a shortened, useless piece of polypeptide chain is produced. The action of the suppressor would be to alter the specificity of the code reading apparatus, possibly by changing the activity of a $s$-RNA fraction, so that an amino-acid is inserted at the nonsense sequence and the polypeptide chain completed. Manney has suggested that the last model is the more likely, and Hawthorne and Friis ( 1964 ) have provided supporting evidence in demonstrating that there is indeed a class of auxotrophic mutations in yeast suppressible by changes in environment, but that this class and the class of mutations suppressible by super-suppressor are mutually exclusive.

If the " nonsense to sense" theory for the action of super-suppressors is accepted, then we may argue that the existence of a cytoplasmically inherited mutation which renders the super-suppressor inactive shows that there must be a self-replicating particle, or a self-sustaining system in the cytoplasm, concerned specifically with the code-reading apparatus. There are several possibilities. One is that these particles are the ribosomes. Another is that they are a system or part of a system, other than chromosomal DNA, responsible for assembling or "priming" transfer RNA. A third might be that they are the transfer RNA molecules themselves. It will obviously be of interest to establish the nature of this cytoplasmic mutation, both from the bearing it might have on the nature of cytoplasmic inheritance in fungi generally, and from the light it might throw on the organisation of polypeptide synthesis in the cell.

\section{SUMMARY}

In some super-suppressed mutant strains of yeast, reversions to the mutant, adenine-requiring, condition were found. The reversions were stable. Genetic analysis established that the revertants still carried the super-suppressor gene, and that the revertant phenotype did not segregate in a Mendelian pattern. It is suggested that the reversion was due to a cytoplasmic mutation, $\psi^{+} \rightarrow \psi^{-}$. This mutation acts to render the super-suppressor inactive, since two suppressible alleles other than the $a d_{2,1}$ allele, are no longer suppressed by supersuppressor in $\psi^{-}$- strains.

\section{REFERENCES}

BENZER, S., AND CHAMPE, S. P. 1962. A change from nonsense to sense in the genetic code. Proc. natl. Acad. Sci. U.S., 48, I I I 4-1121.

CAMPBELL, A. I961. Sensitive mutants of bacteriophage. Virology, 14, 22-32.

Carbondale. 1963. Yeast Genetics Conference. Microbial Genet. Bull., 19 (Suppl.). CoX, B. s., ANd beVAn, E. A. 1962. Aneuploidy in yeast. New. Phytol., 6 $1,342-355$. DARLINGTON, C. D. 1944. Heredity, development and infection. Nature, 154, I64-169. 
EPHRUSSI, B. 1953. Nucleo-Cytoplasmic Relations in Micro-organisms. Clarendon Press, Oxford.

FOWELL, R. R. 1951. Sodium acetate agar as a sporulation medium for yeasts. Nature, 170,587 .

HAWTHORNE, D. C., AND FRIS, J. 1964. Osmotic remedial mutants. A new classification for nutritional mutants in yeast. Genetics, 50, 829-839.

HAWTHORNE, D. C., AND MORTIMER, R. K. 1963. Super-suppressors in yeast. Genetics, $48,617-620$.

JiNks, J. L. 1963. Extra-chromosomal Inheritance. Prentice-Hall Inc., New Jersey.

JiNKs, J. L. 1964. Somatic segregation of extrachromosomally inherited differences in fungi. Proc. XI Internat. Congress Genet., Pergamon Press, pp. 567-577.

JOHNSTON, J. R., AND MORTIMER, R. K. 1959. Use of snail digestive juice in isolation of yeast spore tetrads. F. Bact., 78, 292.

Lindegren, c. c. 1949. The reast Cell: Its Genetics and Cytology. Educational Publishers, Inc., St Louis.

MANNEY, T. R. 1964. Action of a super-suppressor in yeast in relation to allelic mapping and complementation. Genetics, 50, I09-1 21 .

NOVICK, A., AND WEINER, M. 1957. Enzyme induction as an all or none phenomenon. Proc. natl. Acad. Sci. U.S., 47, 1588.

PERKINS, D. D. 1953. The detection of linkage in tetrad analysis. Genetics, 38 , $187-197$.

ROMAN, H. 1956. A system selective for mutations affecting the synthesis of adenine in yeast. C. R. Lab. Carlsberg. Ser. Physiol., 26, 299-314.

SARABhai, A. s., stretton, A. O. W., BRenNer, s., AND Bolls, A. 1964. Colinearity of the gene with the polypeptide chain. Nature, 201, 13-17.

SUSKIND, S. R., AND KUREK, L. I. 1959. On a mechanism of suppressor gene regulation of tryptophan synthetase activity in Neurospora crassa. Proc. natl. Acad. Sci. U.S., 45, 193-196.

woods, R. A. 1963. D. Phil. Thesis, Oxford. 